\title{
Dynamical excitonic effects in metals and semiconductors
}

\author{
Andrea Marini ${ }^{1}$ and Rodolfo Del Sole ${ }^{2}$ \\ 1 Departamento de Física de Materiales, Universidad del Pais Vasco, \\ and Donostia International Physics Center. E-20018 San Sebastián, Spain \\ ${ }^{2}$ Istituto Nazionale per la Fisica della Materia e Dipartimento di Fisica dell'Università di Roma "Tor Vergata", \\ Via della Ricerca Scientifica, I-00133 Roma, Italy
}

(Dated: November 15, 2018)

\begin{abstract}
The dynamics of an electron-hole pair induced by the time-dependent screened Coulomb interaction is discussed. In contrast to the case where the static electron-hole interaction is considered we demonstrate the occurrence of important dynamical excitonic effects in the solution of the BetheSalpeter equation. This is illustrated in the calculated absorption spectra of noble metals (copper and silver) and silicon. Dynamical corrections strongly affect the spectra, partially canceling dynamical self-energy effects and leading to good agreement with experiment.
\end{abstract}

PACS numbers: 71.35.-y ; 71.10.-w; 78.40.-q

The dynamics of an electron-hole pair in a many body system is a strategic field of research with applications to many different aspects of solid state physics [1]. For a large number of systems the observed light-absorption spectra largely deviate from independent-particle calculations 2]. When carried out including self-energy effects, spectra calculated for semiconductors and insulators are characterized by a shift toward high energies with respect to experiment. Those deviations are corrected by including the electron-hole interaction 2]. The strength of these modifications increases as the inverse of the dielectric constant of the system. In insulators, where the electron-hole interaction is only weakly screened, sharp peaks with energy below the optical gap (bound excitons) can be observed in the experimental spectra [3]. If this description is extrapolated to the metallic case the natural conclusion is that the electron-hole interaction has a negligible effect on the optical spectra of metals as the static electron-hole interaction is completely screened by the long-range part of the dielectric function. This simple argument has been considered definitive to assert that there are no excitonic effects in metals [4]. In silver and copper, however, independent-particle calculations overestimate the experimental absorption spectra strengths by $\sim 30 \%[\underline{5}, 6,6]$, a deviation that may be explained only in terms of many-body effects beyond the independent-particle approximation.

The standard approach to account for the electronhole interaction in optical spectra is based on the solution of the Bethe-Salpeter equation (BSE) 1, 8] for the two-particle Green's function. An important ingredient of the BSE is the electron-hole interaction, described by the screened, time-dependent Coulomb interaction $W\left(\mathbf{r}, \mathbf{r}^{\prime} ; t-t^{\prime}\right)$. However, the BSE with a timedependent interaction is hardly solvable $[9]$ and, as common practice, the electron-hole interaction is assumed to be instantaneous; this is equivalent to approximating the time Fourier transform of $W$ with its static value, $W\left(\mathbf{r}_{1}, \mathbf{r}_{2}, \omega=0\right)$. This approximation is verified a posteriori through the comparison with the experiment and physically corresponds to the assumption that the electron-hole scattering time is much longer than the characteristic screening time of the system (roughly speaking, the inverse of the plasma frequency). Indeed, the static approximation is expected to work well for transition energies much smaller than the plasma frequency [2]. However the most striking examples of systems that do not fulfill this condition are silver and copper. The well-known sharp plasmon of silver, which dominates the electron-energy-loss spectra (EELS) 6, 7], is located just above the interband gap $(\sim 3.9 \mathrm{eV})$. Similarly, the EELS of copper shows strong, broad peaks in the optical range [5].

In this letter we prove that dynamical excitonic effects in copper and silver are possible when the BSE is solved with a time-dependent electron-hole interaction. Their experimental optical spectra are correctly explained only thanks to a delicate interplay between dynamical excitonic and self-energy effects. In order to clarify the relation between the present approach and the previous results obtained within the static approximation [2], we show that the optical spectrum of silicon can be obtained without using the approximations commonly employed in the static approach, that is without neglecting quasiparticle renormalization factors and dynamical electron-hole interaction effects.

The absorption spectrum is given by the imaginary part of the dielectric function $\epsilon(\omega) \equiv 1-8 \pi \vec{\Lambda}^{\dagger} \mathbf{P}(\omega) \vec{\Lambda}$, where $\mathbf{P}(\omega)$ is the matrix representation of the polarization function in the non-interacting electron-hole basis and $\vec{\Lambda}$ is a vector embodying the corresponding optical oscillators. The single-particle states are calculated by means of density-functional-theory (DFT) in the local-density-approximation (LDA) [10], while quasiparticle (QP) corrections are added on top of the DFTLDA band structure following the implementation of the GW method 11] described in Ref. 12]. The polarization function is obtained by solving the BSE, an integral 
equation for the four point electron-hole Green's function $\mathbf{L}\left(t_{1}, t_{2} ; t_{3}, t_{4}\right)$. As we are interested in the polarization function $\mathbf{P}(t) \equiv-i \mathbf{L}(t, 0 ; t, 0)$, the BSE can be rewritten as [13]:

$$
\begin{aligned}
& \mathbf{P}(t)=\mathbf{P}^{(0)}(t)-\int d t_{1} \mathbf{P}^{(0)}\left(t-t_{1}\right) \mathbf{V} \mathbf{P}\left(t_{1}\right) \\
& +\iint d t_{1} d t_{2} \mathbf{L}^{(0)}\left(t, t_{2} ; t, t_{1}\right) \widetilde{\mathbf{W}}\left(t_{1}-t_{2}\right) \mathbf{L}\left(t_{1}, 0 ; t_{2}, 0\right),
\end{aligned}
$$

where the BSE kernel is decomposed into a sum of the instantaneous bare electron-hole and exchange interaction $\mathbf{V}$ and of the time dependent screening contribution $\widetilde{\mathbf{W}}: \mathbf{W}(t)=\mathbf{V} \delta(t)+\widetilde{\mathbf{W}}(t)$. Here we have explicitly quoted only time variables, since time dependence is the main concern of this work. $\mathbf{L}^{(0)}$ is the non-interacting electron-hole Green's function whose matrix elements are

$$
\begin{aligned}
& L_{\left(c v \mathbf{k}, c^{\prime} v^{\prime} \mathbf{k}^{\prime}\right)}^{(0)}\left(t_{1}, t_{2} ; t_{3}, t_{4}\right)=\delta_{v, v^{\prime}} \delta_{c, c^{\prime}} \delta_{\mathbf{k}, \mathbf{k}^{\prime}} Z_{c \mathbf{k}} Z_{v \mathbf{k}} \\
& \quad \theta\left(t_{1}-t_{4}\right) e^{-i E_{c \mathbf{k}}\left(t_{1}-t_{4}\right)} \theta\left(t_{3}-t_{2}\right) e^{i E_{v \mathbf{k}}\left(t_{3}-t_{2}\right)},
\end{aligned}
$$

$c, v, \mathbf{k}$ being conduction, valence band and k-point indexes. $E_{n \mathbf{k}}$ (with $n=c, v$ ) and $Z_{n \mathbf{k}}$ (smaller than 1) are the $\mathrm{QP}$ energies and renormalization factors, respectively. The latter represent the weights of the QP peak in the many-body single-particle spectral function. The more $Z_{n \mathbf{k}}$ differs from 1 , the more the high energy structures in the spectral function (like plasmonic replicas) become important, due to the coupling of the QP with system excitations. Those high-energy peaks are not visible in the optical energy range but, nevertheless, subtract intensity from the QP peaks. Very little is known about the role played by the $Z$ factors in optical spectra calculations [14]. If the $Z$ factors are included in an independent-QP calculation [16], or even in the BSE (see below), the intensity of the resulting spectra is strongly underestimated, both in metals and in semiconductors. Thus the $Z$ factors are commonly set to 1 by hand in the solution of the BSE or in the calculation of the independent-QP spectra. This is, again, an approximation, needed to reproduce the experimental results, without a sound theoretical justification.

Because of the time dependent term $\widetilde{\mathbf{W}}\left(t_{1}-t_{2}\right)$, Eq. (1) cannot be rewritten in terms of $\mathbf{P}(t)$ only. For this reason the $\mathrm{BSE}$ is considered hardly solvable (if not "practically unsolvable" 14]) and for computational convenience the electron-hole interaction is approximated as static, $\widetilde{\mathbf{W}}(t) \approx \widetilde{\mathbf{W}}(\omega=0) \delta(t)$ [1, 2]. Thus Eq. (1) can be formally solved by means of a Fourier transform:

$$
\mathbf{P}(\omega)=\mathbf{P}^{(0)}(\omega)-\mathbf{P}^{(0)}(\omega)(\mathbf{V}+\widetilde{\mathbf{W}}) \mathbf{P}(\omega)
$$

This is the static BSE (SBSE) commonly applied neglecting the renormalization factors in Eq. (2), i.e. taking $Z_{n \mathbf{k}}=1$. It yields optical spectra in good agreement with experiments in semiconductors and insulators [2]. When applied to copper and silver however, the SBSE result (dotted lines in Fig 2) is indistinguishable from the independent-QP calculation, without improving the agreement with experiment. The inclusion of the appropriate $\mathrm{Z}$ factors in the independent-QP polarization, $\mathbf{P}^{0}$, leads to strongly underestimated absorption spectra in all cases [15]. Hence, we look for a solution of equation (1) without the two major approximations employed in the SBSE, i.e. keeping the Z's smaller than 1 and $W$ frequency dependent. To this end we expand $\mathbf{L}^{(0)} \widetilde{\mathbf{W}} \mathbf{L}$ in powers of $\widetilde{\mathbf{W}}$. Using generalized indexes $\mathbf{K}:=(c v \mathbf{k})$ the first order term of this expansion, $\mathbf{P}^{(1)}(t)$, is given by:

$$
\begin{aligned}
& P_{\mathbf{K}_{1} \mathbf{K}_{2}}^{(1)}(t)=\iint d t_{1} d t_{2} \theta\left(t_{1}-t_{2}\right) \\
& \quad\left[L_{\mathbf{K}_{1}}^{(0)}\left(t, t_{2} ; t, t_{1}\right) \widetilde{W}_{\mathbf{K}_{1} \mathbf{K}_{2}}\left(t_{1}-t_{2}\right) L_{\mathbf{K}_{2}}^{(0)}\left(t_{1}, 0 ; t_{2}, 0\right)\right. \\
& \left.+L_{\mathbf{K}_{1}}^{(0)}\left(t, t_{1} ; t, t_{2}\right) \widetilde{W}_{\mathbf{K}_{1} \mathbf{K}_{2}}\left(t_{2}-t_{1}\right) L_{\mathbf{K}_{2}}^{(0)}\left(t_{2}, 0 ; t_{1}, 0\right)\right] .
\end{aligned}
$$

From Eq. (2) it is straightforward to see that

$$
\begin{gathered}
L_{\mathbf{K}_{1}}^{(0)}\left(t, t_{2} ; t, t_{1}\right)=i\left[P_{\mathbf{K}_{1}}^{(0)}\left(t-t_{1}\right) e^{i E_{v_{1} \mathbf{k}_{1}}\left(t_{1}-t_{2}\right)} \theta\left(t_{1}-t_{2}\right)\right. \\
\left.+P_{\mathbf{K}_{1}}^{(0)}\left(t-t_{2}\right) e^{-i E_{c_{1} \mathbf{k}_{1}}\left(t_{2}-t_{1}\right)} \theta\left(t_{2}-t_{1}\right)\right], \\
L_{\mathbf{K}_{2}}^{(0)}\left(t_{1}, 0 ; t_{2}, 0\right)=i\left[P_{\mathbf{K}_{2}}^{(0)}\left(t_{2}\right) e^{-i E_{c_{2} \mathbf{k}_{2}}\left(t_{1}-t_{2}\right)} \theta\left(t_{1}-t_{2}\right)\right. \\
\left.+P_{\mathbf{K}_{2}}^{(0)}\left(t_{1}\right) e^{i E_{v_{2} \mathbf{k}_{2}}\left(t_{2}-t_{1}\right)} \theta\left(t_{2}-t_{1}\right)\right],
\end{gathered}
$$

that inserted in Eq. (4), casts $\mathbf{P}^{(1)}(t)$ as a time convolution of three terms (as shown diagrammatically in Fig 1. left diagram).
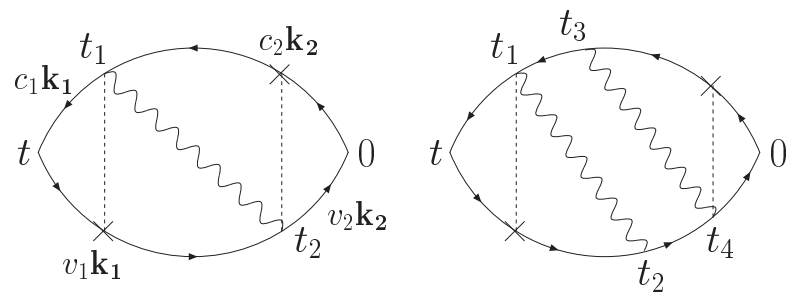

FIG. 1: Diagrammatic representation of the first $\left(\mathbf{P}^{(1)}(t)\right.$ for $t_{1}>t_{2}$, left diagram) and second $\left(\mathbf{P}^{(2, a)}(t)\right.$, right diagram) order contributions to the polarization function $\mathbf{P}(t)$ according to the BSE (see text). Crosses indicate the time points where the incoming and outgoing non-interacting Green's functions are "cut" according to Eqs. (5.6).

As a consequence, in the frequency domain $\mathbf{P}^{(1)}(\omega)$ has the form:

$$
\mathbf{P}^{(1)}(\omega)=-\mathbf{P}^{(0)}(\omega)\left[\boldsymbol{\Pi}^{(a)}(\omega)+\boldsymbol{\Pi}^{(b)}(\omega)\right] \mathbf{P}^{(0)}(\omega),
$$


with $\Pi_{\mathbf{K}_{1} \mathbf{K}_{2}}^{(a)}(\omega)=\widetilde{W}_{\mathbf{K}_{1} \mathbf{K}_{2}}^{(+)}\left(\omega+E_{v_{1} \mathbf{k}_{1}}-E_{c_{2} \mathbf{k}_{2}}\right)$ and $\Pi_{\mathbf{K}_{1} \mathbf{K}_{2}}^{(b)}(\omega)=\widetilde{W}_{\mathbf{K}_{1} \mathbf{K}_{2}}^{(+)}\left(\omega+E_{v_{2} \mathbf{k}_{2}}-E_{c_{1} \mathbf{k}_{1}}\right), \widetilde{\mathbf{W}}^{(+)}(\omega)$ being the Laplace transform of $\widetilde{\mathbf{W}}(t)$. The two terms denoted by $(a)$ and $(b)$ correspond to the two possible time orderings of the interaction ends $\left(t_{1}>t_{2}\right.$ for term (a), shown in Fig प $t_{2}>t_{1}$ for term (b), not shown). Eq. (7) can be thought of as the first order expansion of $\mathbf{P}(\omega)$ in the frequency-dependent interaction $\boldsymbol{\Pi}(\omega)=\boldsymbol{\Pi}^{(a)}(\omega)+\boldsymbol{\Pi}^{(b)}(\omega)$, which replaces $\widetilde{\mathbf{W}}$ of the SBSE. Thus a partial summation of the BSE can be performed writing:

$$
\mathbf{P}(\omega)=\mathbf{P}^{(0)}(\omega)-\mathbf{P}^{(0)}(\omega)[\mathbf{V}+\boldsymbol{\Pi}(\omega)] \mathbf{P}(\omega) .
$$

This is the Dynamical Bethe-Salpeter equation (DBSE), the central result of this work. The diagrams summed up in Eq. (8) are those containing the ladder series of repeated electron-hole interactions with non overlapping (in time) interaction lines. The poles of $\mathbf{P}(\omega), \varnothing_{\lambda}$, will be given by the solution of the equation $\left[\mathbf{P}^{(0)}\left(\varnothing_{\lambda}\right)\right]^{-1}+$ $\mathbf{V}+\boldsymbol{\Pi}\left(\varnothing_{\lambda}\right)=0$. In contrast to the kernel of the SBSE, $\Pi\left(\varnothing_{\lambda}\right)$ is not hermitian and, consequently, $\varnothing_{\lambda}$ is in general complex. Its imaginary part gives the inverse excitonic lifetime. Thus the interacting electronhole states are actually dressed excitons, or quasiexcitons. This agrees with what has been already found in the core exciton limit [9] and emphasizes the analogy between the DBSE and the Dyson equation. Consequently, as in the single-particle problem, we expect to find similar renormalization effects on the quasiexcitonic Green's function. To develop further this aspect we expand linearly the smooth function $\widetilde{\mathbf{W}}^{(+)}(\omega)$ around the non-interacting electron-hole energies, obtaining $\Pi_{\mathbf{K}_{1} \mathbf{K}_{2}}(\omega) \approx \Pi_{\mathbf{K}_{1} \mathbf{K}_{2}}^{(s t)}+\Theta_{\mathbf{K}_{1} \mathbf{K}_{2}}\left(\omega-E_{c_{2} \mathbf{k}_{2}}+E_{v_{2} \mathbf{k}_{2}}\right)$. $\Pi_{\mathbf{K}_{1} \mathbf{K}_{2}}^{(s t)}=\left.\Pi_{\mathbf{K}_{1} \mathbf{K}_{2}}(\omega)\right|_{\omega=E_{c_{2} \mathbf{k}_{2}}-E_{v_{2} \mathbf{k}_{2}}}$ is the static limit of the dynamical Bethe-Salpeter kernel which turns out to be quite similar to the kernel of the SBSE. $\Theta_{\mathbf{K}_{1} \mathbf{K}_{2}}=\partial \Pi_{\mathbf{K}_{1} \mathbf{K}_{2}}(\omega) /\left.\partial \omega\right|_{\omega=E_{c_{2} \mathbf{k}_{2}}-E_{v_{2} \mathbf{k}_{2}}}$ are the excitonic dynamical-renormalization factors. Thus Eq. (8) can be strongly simplified in the case of noble metals where the effect of $\boldsymbol{\Pi}^{(s t)}+\mathbf{V}$ is very small. The corresponding polarization function $\mathbf{P}(\omega)$ is approximatively given by:

$$
P_{\mathbf{K}_{1} \mathbf{K}_{2}}(\omega) \approx \frac{\left[\left(\mathbf{Z}^{e h}\right)^{-1}+\boldsymbol{\Theta}\right]_{\mathbf{K}_{1} \mathbf{K}_{2}}^{-1}}{\omega-E_{c_{2} \mathbf{k}_{2}}+E_{v_{2} \mathbf{k}_{2}}+i 0^{+}},
$$

with $Z_{\mathbf{K}_{1} \mathbf{K}_{2}}^{e h}=Z_{c_{1} \mathbf{k}_{1}} Z_{v_{1} \mathbf{k}_{1}} \delta_{\mathbf{K}_{1} \mathbf{K}_{2}}$. The connection between dynamical excitonic and self-energy effects is now clear. $Z_{n \mathbf{k}}^{-1}=1-\beta_{n \mathbf{k}}$, where the negative factor $\beta_{n \mathbf{k}}$, the frequency derivative of the self-energy, is the weight lost by the QP because of the coupling with the excitations of $W(\omega)$. The excitonic factors $\boldsymbol{\Theta}$, instead, are due to the modification of such coupling as a consequence of the electron-hole interaction. Those two effects tend to cancel each other but the cancellation is, in general, not complete, as exemplified in Fig 2 for copper and silver. The SBSE calculation (dotted line), with $Z_{n \mathbf{k}}=1$ and $\Theta=0$, overestimates the experimental intensity (circles), while the inclusion of the $Z$ 's only (dashed line) underestimates it [7]. In the DBSE (full line), obtained solving Eq. (8), the dynamical $\Theta$ factors partially compensate for the $\mathbf{Z}^{e h}$ factors yielding a spectral intensity in good agreement with experiment.
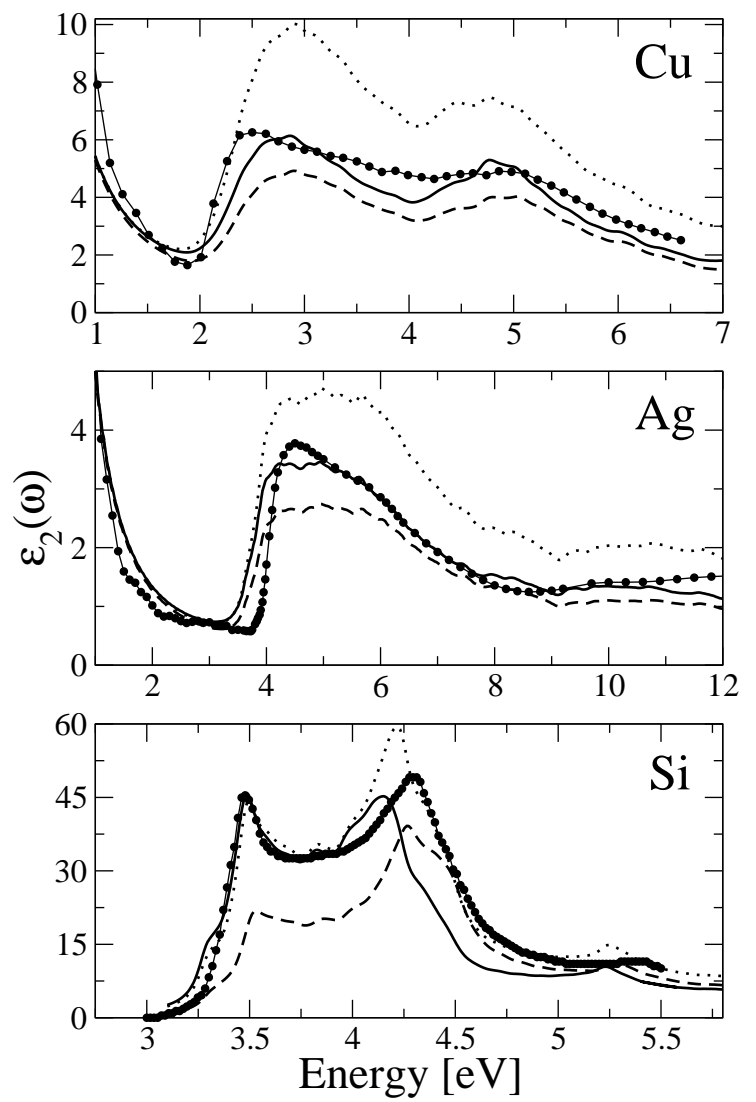

FIG. 2: Absorption spectrum of bulk copper, silver, and silicon. Dotted line: SBSE without renormalization factors. Dashed line: SBSE including the QP renormalization factors. Full line: result of the dynamical Bethe-Salpeter equation including dynamical QP and excitonic effects. Circles: experimental spectra (Ref. 17] for $\mathrm{Cu}$ and $\mathrm{Ag}$ and Ref. [18] for $\mathrm{Si}$ ).

The case of bulk silicon is also shown in Fig 2 bottom panel. The SBSE spectrum, calculated in the usual way by setting the $Z$ factors to 1 by hand (dotted line) is in good agreement with the experiment (circles). On the other hand, the spectrum including dynamical factors in the SBSE (dashed line) underestimates the experiment, as anticipated above, and the relative intensity of the two peaks is only poorly reproduced. The solution of the DBSE (solid line), instead, is in good agreement with the experiment. In contrast to the metallic case, however, the DBSE kernel of silicon must contain second- 
order contributions in order to reproduce correctly the experimental optical spectrum. The main effect of the first order kernel $\Pi(\omega)$ is indeed to balance the reduction of optical strengths due to self-energy renormalization factors, as suggested by Bechstedt et al. [14]. However, the renormalized QP weights also imply a reduction of the statically screened electron-hole of almost $\sim 30 \%$, which is the reason for the wrong relative intensities of the two peaks in the SBSE result. This shortcoming is fixed by the second-order diagrams, as discussed below. One of the two second order diagrams, $\mathbf{P}^{(2, a)}(t)$, is shown in Fig[1] right diagram. In the other second-order diagram $\left(\mathbf{P}^{(2, b)}(t)\right.$, not shown), the interaction lines are ordered according to $t>t_{2}>t_{4}>t_{1}>t_{3}>0$. Using Eqs. (5.6) we can isolate the external non-interacting polarization functions (as schematically shown in Fig 1 by the crosses corresponding to the time points $t_{1}$ and $\left.t_{4}\right)$. Consequently $\mathbf{P}^{(2, a)}(t)$ can be Fourier transformed to yield $\mathbf{P}^{(2, a)}(\omega)=-\mathbf{P}^{(0)}(\omega) \boldsymbol{\Pi}^{(2, a)}(\omega) \mathbf{P}^{(0)}(\omega)$ with $\boldsymbol{\Pi}^{(2, a)}(\omega)=\boldsymbol{\Theta}^{(a)} \mathbf{Z}^{e h} \boldsymbol{\Pi}^{(a)}(\omega)$. Together with the contribution from diagram $\mathbf{P}^{(2, b)}$, we obtain the total secondorder kernel,

$$
\boldsymbol{\Pi}^{(2)}(\omega)=\boldsymbol{\Pi}(\omega)+\sum_{s=a, b} \boldsymbol{\Theta}^{(s)} \mathbf{Z}^{e h} \boldsymbol{\Pi}^{(s)}(\omega)
$$

The solution of the DBSE with the kernel given by Eq. (10) is equivalent to summing all diagrams of the original BSE with up to two overlapping interaction lines. While the first-order term $(\boldsymbol{\Pi}(\omega))$ partially restores the optical-strength intensities, the second-order correction $\left(\boldsymbol{\Theta}^{(s)} \mathbf{Z}^{e h} \boldsymbol{\Pi}^{(s)}(\omega)\right)$ reduces the screening of the electron-hole pair, thus enhancing their interaction. In the case of silicon this effect improves considerably the relative intensity of the two main peaks, as shown in Fig.2(continuous line). Higher-order contributions can also be included in the DBSE kernel, although these terms contain either higher powers of $\boldsymbol{\Theta}$ or higher order energy derivatives of $\Pi(\omega)$ [19] that are negligible in the cases studied in this work.

Thus, in the case of copper and silver the BSE must be solved using a time-dependent electron-hole interaction to obtain good agreement with the experiment. The DBSE result in the case of silicon is similar to the case when, in the SBSE, the QP renormalization factors are neglected [2]. We understand this result as follows: the electron-hole pair, being a neutral excitation, is less efficient than the electron and the hole alone in exciting virtual plasmons, which is the main process leading to QP renormalization. Only when dynamical effects are coherently included both in the self energy and in the electron-hole interaction, this (physically expected) result emerges from the bundle of many-body equations. This confirms the SBSE results but not the separate approximations involved therein. Even though the plasma frequency of silicon is at $\sim 16 \mathrm{eV}$ (far above the optical energy range), the DBSE kernel needs a second order contribution to balance the self-energy dynamical factors.

In conclusion, we have shown that, in contrast to common belief, dynamical excitonic effects in metals do exist and are crucial for reproducing the experimental optical absorption. We have demonstrated that dynamical excitonic and self-energy effects must be included together in the calculation of the response functions as they do not completely cancel each other. The good agreement with experiment obtained using a static BSE kernel for semiconductors is confirmed by the present, more general approach.

This work has been supported by the the EU through the NANOPHASE Research Training Network (Contract No. HPRN-CT-2000-00167). We also acknowledge support from INFM PAIS CELEX and from MIUR Cofin 2002. We thank A. Rubio and F. Bechstedt for helpful discussions, and C. Hogan for a critical reading.

[1] For a review, see G. Onida, L. Reining, and A. Rubio, Rev. Mod. Phys. 74, 601 (2002).

[2] S. Albrecht, L. Reining, R. Del Sole, and G. Onida, Phys. Rev. Lett. 80, 4510 (1998). L.X. Benedict, E.L. Shirley, and R.B. Bohn, ibid. 80, 4514 (1998); M. Rohlfing and S.G. Louie, ibid. 81, 2312 (1998).

[3] see, for instance, D. M. Roessel and W. C. Walker, J. Opt. Soc. Am. 57, 835 (1967) for the case of LiF.

[4] The existence of excitonic effects in metals on an ultrashort time scale has been speculated by W.D. Schone and W. Ekardt Phys. Rev. B 62, 13464 (2000); 65, 113112 (2002). Those transient excitons, however, cannot be detected with traditional photoabsorption techniques.

[5] A. Marini, R. Del Sole, G. Onida, Phys. Rev. B 64, 195125 (2001).

[6] A. Marini, R. Del Sole, G. Onida, Phys. Rev. B 64, 115101 (2002).

[7] M. A. Cazalilla, J. S. Dolado, A. Rubio, and P. M. Echenique, Phys. Rev. B 61, 8033 (2000). V. P. Zhukov, F. Aryasetiawan, E. V. Chulkov, I. G. de Gurtubay, and P. M. Echenique, ibid. 64, 195122 (2001).

[8] G. Strinati, Rivista del nuovo cimento, 11, 1 (1988).

[9] G. Strinati, Phys. Rev. Lett. 49, 1519 (1982); Phys. Rev. B 29, 5718 (1984).

[10] DFT calculations are performed using a plane-wave basis and norm-conserving pseudopotentials (PP) [N. Troullier and J.L. Martins, Phys. Rev. B 43, 1993 (1991)]. The use of soft PPs, in the case of $\mathrm{Ag}$ and $\mathrm{Cu}$, allowed us to work with an energy cut-off of $60 \mathrm{Ry}[5,6]$.

[11] L. Hedin, Phys. Rev. 139, A796 (1965). For a review, see F. Aryasetiawan and O. Gunnarsson, Rep. Prog. Phys. 61, 237-312 (1998).

[12] A. Marini, R. Del Sole, G. Onida, Phys. Rev. Lett. 88, 016403 (2002).

[13] For simplicity, only the coupling between the resonant parts of $\mathbf{L}^{(0)}$ are considered. This is an approximation commonly used to solve the SBSE. Here $\mathbf{P}$ is the macroscopic polarization indicated as $\overline{\mathbf{P}}$ in Ref. [1]. Correspondingly, the electron-hole exchange interaction, embodied 
in $\mathbf{V}$, does not contain the $\mathbf{G}=0$ component.

[14] Neglecting the static excitonic effects (corresponding to $\boldsymbol{\Pi}^{(s t)}=0$ in the DBSE), an almost complete cancellation between dynamical quasiparticle and excitonic effects in silicon and diamond, at the first order of the BSE has been demonstrated by F. Bechstedt, K. Tenelsen, B. Adolph, and R. Del Sole, Phys. Rev. Lett. 78, 1528 (1997).

[15] Following Ref. 6, 12] we include 3s/3p (4s/4p) core levels in the GW self-energy of copper (silver). Even if those states drastically modify the QP energies, they leave almost unchanged the $Z$ factors, and consequently, the ab- sorption intensity.

[16] R. Del Sole and R. Girlanda, Phys. Rev. B 54, 14376 (1996).

[17] E.D. Palik, Handbook of Optical Constants of Solids (Academic Press, New York, 1985).

[18] P. Lautenschlager, M. Garriga, L. Vina, and M. Cardona, Phys. Rev. B 36, 4821 (1987).

[19] We have also calculated the two third-order diagrams $\boldsymbol{\Pi}^{(3, s)}$ containing three overlapping interaction lines finding, respectively, $\boldsymbol{\Pi}^{(3, s)} \sim\left(\boldsymbol{\Theta}^{(s)}\right)^{2}$ and $\boldsymbol{\Pi}^{(3, s)} \sim \frac{\partial^{2} \Pi^{(s)}}{\partial \omega^{2}}$. 